CLINICAL STUDY

\title{
Altered urinary excretion of aquaporin 2 in IgA nephropathy
}

Maria Teresa Rocchetti, Grazia Tamma ${ }^{1}$, Domenica Lasorsa ${ }^{1}$, Ida Valentina Suriano, Annamaria D’Apollo, Massimo Papale, Lisa Mastrofrancesco ${ }^{1}$, Giuseppe Grandaliano, Maria Svelto ${ }^{1}$, Giovanna Valenti ${ }^{1}$, Loreto Gesualdo ${ }^{2}$ and Salvatore Di Paolo ${ }^{3}$

Section of Nephrology and Bioagromed, Department of Biomedical Sciences, University of Foggia, 71122 Foggia, Italy, ${ }^{1}$ Department of General and Environmental Physiology, University of Bari, 70126 Bari, Italy, ${ }^{2}$ Section of Nephrology, Department of Internal Medicine and Public Health, DiMIMP, University of Bari, Piazza G. Cesare, 11, 70124 Bari, Italy and ${ }^{3}$ Division of Nephrology and Dialysis, Hospital 'Dimiccoli', 70051 Barletta, Italy

(Correspondence should be addressed to L Gesualdo; Email: l.gesualdo@nephro.uniba.it)

\begin{abstract}
Objective: The intrarenal renin-angiotensin system (RAS) activation plays a pivotal role in immunoglobulin A nephropathy (IgAN) pathogenesis, which is still largely undefined. Recently, vasopressin (AVP) has been advocated to contribute to the genesis and progression of chronic kidney diseases (CKD) directly, and indirectly, via RAS activation. Our aim is to explore the intrarenal activity of AVP, its relationship with RAS activity, as well as its modulation by therapies in $\operatorname{IgAN}$.

Design: In this observational study, we measured plasma copeptin, a surrogate marker of AVP, the urine excretion of aquaporin 2 (AQP2), a protein reflecting renal AVP action, and angiotensinogen (AGT), a parameter of renal RAS activation, and their relationship with renal function in $44 \mathrm{IgAN}$ patients at the time of renal biopsy, without any drug therapy, and after 6-month treatment with ACEi or steroid + ACEi. Twenty-one patients with other CKD and 40 healthy subjects were recruited as controls.

Methods: ELISAs were used to measure all variables of interest.

Results: At baseline, IgAN patients showed higher urinary levels of AQP2, compared with controls and patients with other CKD. Urinary AQP2 and AGT levels strongly correlated with the presence of arterial hypertension. Steroids + ACEi caused the decrease of all the variables examined. The fall of urinary AQP2 and AGT following drug treatments was associated with the decrease of daily proteinuria.

Conclusion: Our findings would support the involvement of AVP-AQP2 axis, interacting with the RAS, in the progression of IgAN and candidate AQP2 as a possible novel marker of the disease.
\end{abstract}

European Journal of Endocrinology 165 657-664

\section{Introduction}

Immunoglobulin A nephropathy (IgAN) is now recognised to be the most common form of glomerulonephritis worldwide. Although the mechanisms underlying the pathogenesis and development of IgA nephropathy remain largely undefined, it is gradually being clarified that intrarenal renin-angiotensin system (RAS) activation plays a pivotal role in the pathogenesis and development of the disease $(1,2)$. Accordingly, RAS blockade appears to limit proteinuria and reduce glomerular filtration rate decline more effectively than other antihypertensive treatments in $\operatorname{IgAN}$ patients $(3,4)$. Full remission of the disease, however, is seldom achieved, especially when pharmacological intervention is started late, and supports the adoption of more complex strategies than with an isolated pharmacological intervention on the RAS.

In addition to the RAS, an accumulating body of evidence suggests that arginin-vasopressin (AVP) may play a role in the genesis and exacerbation of renal damage and chronic renal insufficiency (5). Particularly, Bankir (6) argued that the activation of AVP specific G protein-coupled $\mathrm{V} 2$ renal receptors, by regulating aquaporin 2 (AQP2) expression and subsequent insertion into the luminal membrane of collecting duct principal cells, increases urea recycling from the collecting duct into the loop of Henle; reduces sodium concentration at the macula densa; inhibits tubuloglomerular feedback; stimulates renin release and could in this way result in glomerular hyperfiltration, proteinuria and renal damage $(6,7)$.

Then, several findings suggest the existence of a crosstalk between AVP and the RAS. Angiotensin II (Ang II) potently induces AQP2 protein expression via AVP V2 and Ang II AT(1) receptors in the mouse renal collecting duct principal cells (8), while a sustained stimulation of AVP receptors induces intrarenal RAS activation $(5,6)$. Conversely, RAS blockade decreases AQP2 expression (8) and blunts AVP-induced rise in proteinuria in experimental models (7). 
Table 1 Demographic and laboratory features of healthy controls, IgAN patients (before and after 6-month treatment), and patients with chronic kidney diseases other than IgAN. Data are expressed as mean \pm S.D.

\begin{tabular}{|c|c|c|c|c|c|c|c|}
\hline & \multirow{3}{*}{$\begin{array}{l}\text { Healthy } \\
\text { controls } \\
(n=40)\end{array}$} & \multirow[b]{3}{*}{ CKD $(n=21)$} & \multicolumn{5}{|c|}{$\operatorname{IgAN}$} \\
\hline & & & \multirow{2}{*}{$\begin{array}{c}\text { All }(n=44) \\
\text { Baseline }\end{array}$} & \multicolumn{2}{|c|}{ ACEi $(n=25)$} & \multicolumn{2}{|c|}{ Steroids + ACEi $(n=19)$} \\
\hline & & & & Baseline & 6 months & Baseline & 6 months \\
\hline Age (years) & $38 \pm 14$ & $37 \pm 18$ & $40 \pm 14$ & $39 \pm 13$ & - & $45 \pm 14$ & - \\
\hline Sex & $26 \mathrm{M} / 14 \mathrm{~F}$ & $13 \bar{M} / 8 \mathrm{~F}$ & $32 \mathrm{M} / 12 \mathrm{~F}$ & $19 \bar{M} / 6 \mathrm{~F}$ & - & $13 \bar{M} / 6 \mathrm{~F}$ & - \\
\hline Serum creatinine $(\mathrm{mg} / \mathrm{dl})$ & $0.8 \pm 0.2$ & $0.90 \pm 0.32$ & $1.03 \pm 0.36$ & $0.90 \pm 0.27$ & $0.91 \pm 0.21$ & $1.28 \pm 0.38^{*}$ & $1.27 \pm 0.37^{\star}$ \\
\hline $\begin{array}{l}\text { Proteinuria (g/day) } \\
\text { IgAN Histology grading }\end{array}$ & $\begin{array}{l}\text { NA } \\
\text { NA }\end{array}$ & $\begin{array}{c}2.49 \pm 2.93 \\
N A\end{array}$ & $1.59 \pm 1.62$ & $1.07 \pm 0.91$ & $0.64 \pm 0.59$ & $2.39 \pm 2.15^{\dagger}$ & $0.82 \pm 0.89$ \\
\hline G1 & & & & 1 & - & - & - \\
\hline G2 & & & & 9 & - & - & - \\
\hline G3 & & & & 12 & - & 10 & - \\
\hline G4 & & & & 3 & - & 9 & - \\
\hline MDRD GFR $\left(\mathrm{ml} / \mathrm{min}\right.$ per $\left.1.73 \mathrm{~m}^{2}\right)$ & & $102.0 \pm 38.2$ & $78.7 \pm 25.9^{\S}$ & $88.9 \pm 22.9$ & $94.0 \pm 22.2$ & $66.1 \pm 24.4^{\ddagger}$ & $69.3 \pm 27.3^{\dagger}$ \\
\hline Hypertension (yes/not) & - & $4 / 17$ & $21 / 23$ & $8 / 17$ & & $13 / 6$ & \\
\hline
\end{tabular}

${ }^{\star} P<0.05,{ }^{\dagger} P<0.01,{ }^{\ddagger} P<0.005$ : steroids + ACEi versus ACEi alone (Mann-Whitney $U$ test) and ${ }^{\S} P=0.05$ versus CKD.

Presently, no data are available concerning the activity of AVP in IgAN patients. This study was undertaken to explore the intrarenal activity of AVP, its relationship with RAS activity, as well as its modulation by currently available therapies for patients with IgAN. To this aim, we measured the urinary excretion of AQP2 (uAQP2), a shadow protein reflecting renal AVP action (9), and angiotensinogen (uAGT), a newly proposed parameter of renal RAS activation, at the time of histological diagnosis, and following two different therapeutic approaches, currently representing the most adopted first-line treatments: ACEi alone or steroids in combination with ACEi $(3,4)$.

\section{Subjects and methods}

\section{Patients}

Between January 2005 and December 2009, we selected 80 consecutive IgAN patients biopsied at the Section of Nephrology, University of Foggia. Entry criteria were: age $>18$ years, grades I-IV lesions (Lee's classification (10)), absence of nephrotic syndrome, concomitant systemic disease and urological abnormalities and/or infection and plasma creatinine levels of $\leq 2.0 \mathrm{mg} / \mathrm{dl}$. Thirty-six patients, taking RAS blockers at the time of renal biopsy, were excluded, thus 44 IgAN patients were recruited for this study (Table 1). All patients received ACEi, titrated at the maximally tolerated dose (5-10 mg ramipril). Nineteen subjects with moderate to severe daily proteinuria (1.0$8.4 \mathrm{~g} / \mathrm{die})$ and grades III and IV histological lesions were assigned to steroids and received $0.5 \mathrm{~g}$ methylprednisolone i.v. for three consecutive days at the beginning of the steroid course and again 2 and 4 months later; they were also given oral prednisone at a daily dose of $0.5 \mathrm{mg} / \mathrm{kg}$ for 6 months. The remaining
25 patients received ACEi only. Hypertension was defined as blood pressure (BP) of more than $140 / 90 \mathrm{mmHg}$ in two repeated measurements in a standing position, or the need for antihypertensive agents. All patients in both groups were targeted to achieve $\mathrm{BP}$ values $\leq 130 / 80 \mathrm{mmHg}$ by ACEi, and calcium channel blockers if needed. All patients were studied before and after the designed therapy. None of the patients took
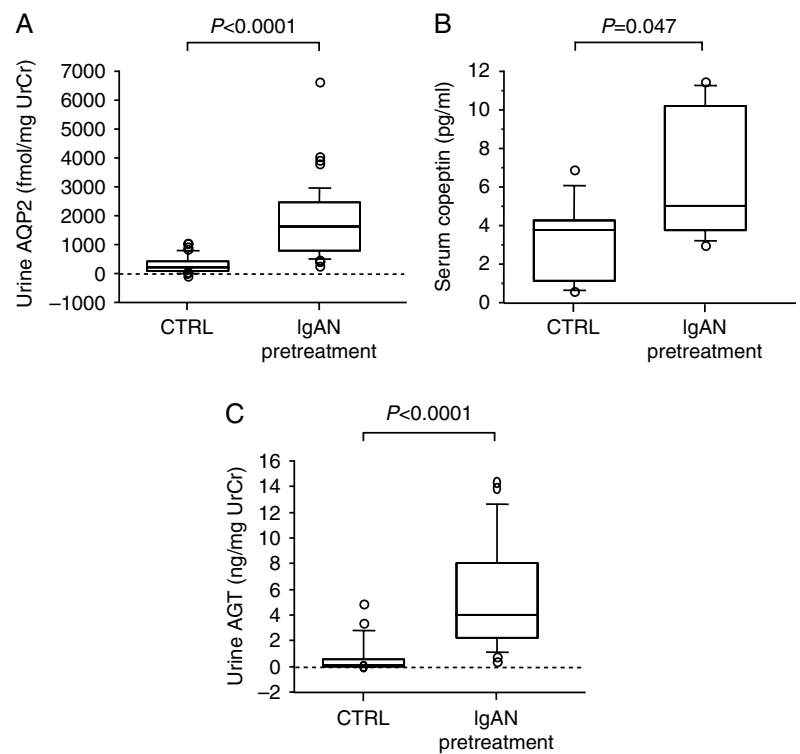

Figure 1 (A) Urinary levels of aquaporin 2 (AQP2), (B) plasma levels of copeptin and (C) urinary levels of angiotensinogen (AGT) in healthy individuals (CTRL), and in patients with IgAN measured at the time of renal biopsy, in the absence of any drug therapy. The boxes extend from the first quartile to the third quartile and the whiskers extend to the 10th and 90th percentiles of the data. Individual values beyond the 10th and 90th percentiles are also shown. The central horizontal line within each box represents the 50th percentile (median) (Mann-Whitney $U$ test). 

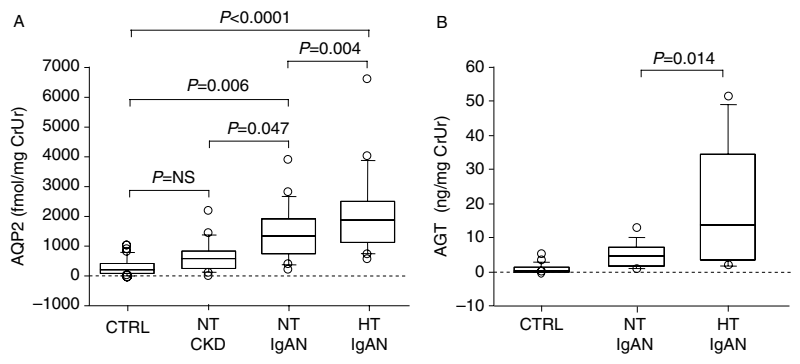

Figure 2 (A) Urinary levels of aquaporin 2 (AQP2) in healthy individuals (CTRL), in normotensive CKD patients, and in IgAN patients, distinguished in normotensives (NT) and hypertensives (HT). (B) Urinary levels of angiotensinogen (AGT) in healthy individuals and in hypertensive and normotensive IgAN patients. All variables were measured at the time of renal biopsy, in the absence of any drug therapy. Box-and-whisker plots, as in Fig. 1 (ANOVA test).

diuretics throughout the study period. We further enrolled 21 patients with chronic kidney disease (CKD) other than IgAN (seven with membranous glomerular nephropathy, eight with focal segmental glomerular sclerosis and six with benign nephroangiosclerosis) (Table 1). All patients were investigated at the time of histological diagnosis. Forty healthy controls without any personal or family history of renal disease and arterial hypertension were enrolled as well. The study was approved by the local ethics committee, and a written informed consent was obtained from all participants. All investigations were conducted according to the principles expressed in the Declaration of Helsinki.

\section{Sample collection}

Blood and first-void urine were collected early in the morning, after an overnight fast, all subjects being on their usual diet, with free access to fluids.

Urine samples were first tested for standard parameters using Multistix reactive stripes (Bayer Diagnostics), then centrifuged and filtered, after the addition of protease inhibitors cocktail (P8340, SigmaAldrich).

Blood samples were collected in BD Vacutainer Rapid Serum tubes and centrifuged.

All processed samples were stored as aliquots at $-80{ }^{\circ} \mathrm{C}$ until use.

\section{Urinary AGT, AQP2, bradykinin and plasma copeptin assays}

Copeptin plasma levels, and bradykinin (BK) and AGT urinary levels were measured using commercially available ELISA kits (BK and copeptin: Uscn Life Science, Inc., Wuhan, China; AGT: Immuno-Biological Laboratories Co., Gunma, Japan). Urinary AQP2 was measured by ELISA as described previously (11). Urine concentration of BK, AGT and AQP2 was expressed as proteinto-urinary creatinine ( $\mathrm{UrCr}$, in $\mathrm{mg}$ ) ratio.

\section{Statistical analysis}

All data are presented as proportions of the patient population, mean \pm s.D. or median and range, as appropriate. Differences between quantitative non-parametric variables were tested by the Mann-Whitney $U$ test and Wilcoxon test, as appropriate. The correlation between non-parametric variables was determined by Spearman's rank correlation test. Adjustment was made for relevant covariates that had been found significant $(P<0.05)$ in univariate analysis. Logistic regression was used to determine factors significantly related to the presence of arterial hypertension and to deterioration of kidney function over time (i.e. change of proteinuria following drug therapies). Variables with a significance level $<0.05$ at simple logistic analysis were next fit in a multivariate model. The risk is expressed as odds ratio (OR) $\pm 95 \%$ confidence interval (CI). The Statview software package, SAS (5.0 version; Cary, NC, USA) was used for all analyses.

\section{Results}

\section{Baseline evaluation}

At the time of histological diagnosis, IgAN patients had significantly higher levels of UAQP2, compared with healthy controls (Fig. 1) as well as to patients with CKD other than IgAN (1964.62 \pm 275.44 vs 720.96 $\pm 114.43 \mathrm{fmol} / \mathrm{mg}$ UrCr; $P=0.0004)$. Vesicular trafficking and long-term changes in the renal abundance of AQP2 water channels are mainly modulated by AVP. Therefore, we next measured copeptin, a marker of endogenous AVP (12), and found that IgAN patients

Table 2 Relationship between systemic hypertension and urinary levels of AQP2 and AGT in IgAN patients, analysed by logistic regression.

\begin{tabular}{|c|c|c|c|c|c|c|c|c|}
\hline & \multicolumn{4}{|c|}{ Simple logistic analysis } & \multicolumn{4}{|c|}{ Multiple logistic analysis } \\
\hline & OR & $95 \% \mathrm{Cl}$ & $P^{*}$ & $R^{2}$ & OR & $95 \% \mathrm{Cl}$ & $P^{*}$ & $R^{2}$ \\
\hline AQP2 (100 fmol/mg Ucr) & 1.22 & $1.02-1.45$ & 0.0014 & 0.262 & 1.16 & $0.93-1.45$ & & t \\
\hline AGT (ng/ml Ucr) & 1.16 & $0.93-1.45$ & 0.0078 & 0.404 & 1.11 & $0.95-1.31$ & & \\
\hline
\end{tabular}

${ }^{*}$ likelihood ratio; ${ }^{\dagger}$ comparing AQP2 and AGT Cl levels. 
Table 3 Effects of drug therapy (ACEi and ACEi+steroids) on renal salt and water handling in the cohort of IgAN patients. Data are presented as median (range).

\begin{tabular}{lccccc}
\hline & \multicolumn{2}{c}{ ACEi } & & \multicolumn{2}{c}{ ACEi + steroids } \\
\cline { 2 - 3 } & Baseline & 6 months & & Baseline & 6 months \\
\hline Plasma sodium (mEq/l) & $139(133-143)$ & $140(134-143)$ & & $138.5(134-143)$ & $139(136-147)$ \\
Plasma osmolarity (mOsm/l) & $291.4(278.8-305.8)$ & $291.1(282.9-300.3)$ & & $291.5(280.4-301.3)$ & $297.4^{*}(285.7-314.6)$ \\
Urine osmolality (mOsm/kg & $4.0(2.9-7.6)$ & $5.1(1.3-8.4)$ & & $4.0(1.9-5.7)$ & $4.3(1.3-6.3)$ \\
per mg urine creatinine) & $1.2(0.5-3.0)$ & $1.1(0.4-1.9)$ & & $1.6(0.4-5.2)$ & $1.7(0.7-3.2)$ \\
FENa (\%) & & &
\end{tabular}

${ }^{\star} P<0.05$ versus baseline.

had a concomitant increase in the plasma levels of the glycopeptide (Fig. 1).

Then, we found significantly higher levels of uAGT, confirming the RAS activation in IgAN patients (Fig. 1).

Daily proteinuria higher than $1 \mathrm{~g}$ was associated with higher levels of uAQP2 $(2739.0 \pm 553.5$ vs $1579.6 \pm 162.5 \mathrm{fmol} / \mathrm{mg}$ UrCr; $P=0.02$ ) and uAGT $(19.6 \pm 5.9$ vs $6.4 \pm 2.7 \mathrm{ng} / \mathrm{mg} \mathrm{UrCr} ; P=0.004)$.

Similarly, IgAN patients with eGFR lower than $60 \mathrm{ml} / \mathrm{min} \quad(n=10)$ showed significantly higher levels of both AQP2 (2397.1 \pm 178.9 vs 1634.1 $\pm 179.7 \mathrm{fmol} / \mathrm{mg}$ UrCr; $P=0.005)$ and uAGT $(36.2$ \pm 8.5 vs $5.7 \pm 1.7 \mathrm{ng} / \mathrm{mg}$ UrCr; $P=0.001$ ).

At the start of the study, in the absence of any therapy, we were unable to find a direct correlation between UAQP2 and uAGT levels.

None of the biomarkers measured resulted to correlate with age or sex of the patients studied.

\section{Hypertension}

IgAN patients presenting with arterial hypertension at the time of histological diagnosis displayed the highest levels of uAQP2 and uAGT (Fig. 2). Simple logistic analysis showed that both the variables examined were highly correlated with the presence of systemic hypertension. The OR associated with a $100 \mathrm{fmol} / \mathrm{mg} \mathrm{UrCr}$ increase in mean $\mathrm{uAQP} 2$ or $1 \mathrm{ng} / \mathrm{mg} \mathrm{UrCr}$ increase in mean uAGT yielded a 22 or $15 \%$ increase in risk for arterial hypertension respectively (Table 2). When UAQP2 and uAGT were fitted in a multiple logistic regression model, both variables maintained their relationship with the presence of hypertension (Table 2).

\section{End-of-study evaluation}

Six-month treatment did not significantly modify plasma osmolarity, fractional sodium excretion and urine osmolality of IgAN patients (Table 3), which would rule out major modifications of renal salt and water handling in a cohort of subjects on a free diet and with free access to fluids. Of note, the change in urine osmolality following drug treatment correlated with the change of UAQP2 $(P=0.007)$. Corticosteroids caused a significant decrease of both the variables examined (Fig. 3). At variance, 6-month treatment with ACEi failed to significantly modify uAGT or UAQP2 (Fig. 3). RAS blockade by ACEi is acknowledged to increase BK concentration (13), and the kinin would directly impair cell surface expression of AQP2 water channel (14). Therefore, we measured uBK at both the start and the end of the study and found that ACEi caused a significant increase of the kinin, but only in the absence of steroids (Fig. 3). This seemingly negates a relevant interfering effect of $\mathrm{BK}$ on the urine excretion of AQP2 in the IgAN patients studied.

Finally, in the whole group of patients, the change of UAGT following pharmacological treatments significantly correlated with the change of UAQP 2 $(\rho=0.502 ; P=0.0013)$.

\section{Proteinuria}

Drug therapy caused $>50 \%$ decrease of daily proteinuria in $45 \%$ patients treated with ACEi alone and in $58 \%$ patients treated with steroids + ACEi. The estimated glomerular filtration rate was stable during the whole observation period (Table 1).

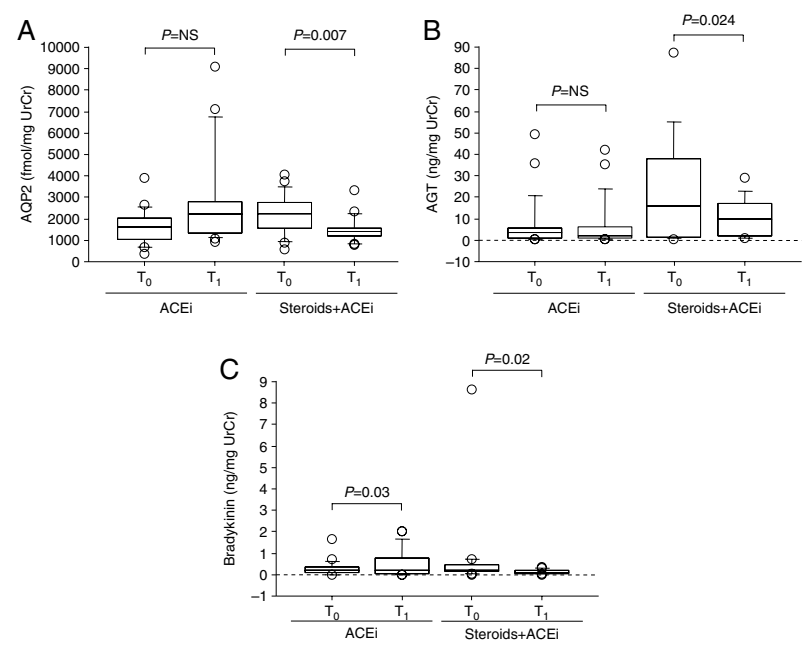

Figure 3 Changes in urinary AQP2 (A), AGT (B) and bradykinin (C) excretion following 6-month treatment with $\mathrm{ACEi}$ alone or steroids + ACEi. Box-and-whisker plots, as in Fig. 1 (Wilcoxon test). 

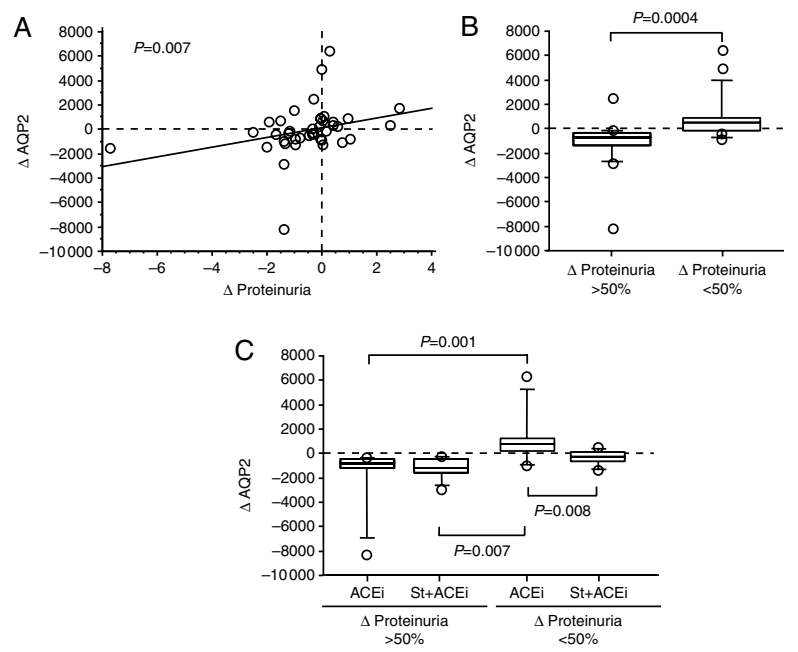

Figure $4(A)$ Correlation between the change $(\Delta)$ of urinary AQP2 and the change $(\Delta)$ of $24 \mathrm{~h}$ proteinuria $(\mathrm{g} / \mathrm{die})$ in IgAN patients after 6-month treatment with either ACEi or steroids + ACEi (Spearman's rank correlation test). (B) Change of AQP2 urine excretion in patients, distinguished in responders $(>50 \%$ decrease of proteinuria) and non-responders ( $<50 \%$ decrease of proteinuria) (Mann-Whitney $U$ test). (C) Change of urinary AQP2 excretion according to the type of treatment and the modification of daily proteinuria (ANOVA test). Box-and-whisker plots, as in Fig. 1.

When patients in both treatment groups were distinguished according to the change of proteinuria at the end of the 6-month period, those with a fall of proteinuria $>50 \%$ displayed also a significant decrease of UAQP2 and uAGT: the higher the decrease of UAQP2 or UAGT, the higher the decrease in protein excretion (Figs 4 and 5). Univariate logistic analysis confirmed that the modifications of UAQP2 and uAGT were independent predictors of the change of proteinuria, regardless of the type of therapy (Table 4 , left panel). When fitted in a multiple logistic regression model, both the independent variables maintained their relationship with the change of proteinuria (Table 4, right panel).

Finally, we tried to ascertain whether the baseline levels of the biomarkers investigated would predict treatment response. Baseline uAQP2 levels, but not serum copeptin or uAGT, were significantly associated with the change of proteinuria recorded at the end of the study $(\rho=-0.489 ; P=0.004)$. Accordingly, patients with higher levels of UAQP2 at baseline showed higher decrements in the urine excretion of the water channel protein following drug therapy $(\rho=-0.745$; $P<0.0001)$.

\section{Discussion}

In this study, we report that at the time of histological diagnosis IgAN patients displayed an early and strong activation of the renal RAS, which was associated with a rise in the renal excretion of the water channel protein AQP2. Furthermore, patients with a greater deterioration of kidney function (i.e. eGFR lower than $60 \mathrm{ml} / \mathrm{min}$ and daily proteinuria higher than $1 \mathrm{~g}$ ) displayed a higher urine excretion of both AGT and AQP2.

Local RAS hyperactivity has long been demonstrated in patients with $\operatorname{IgAN}(2,15)$. Recently, uAGT has been proposed as a reliable marker of activated intrarenal RAS in IgAN: increases in UAGT levels were correlated with augmented intrarenal AGT gene expression and Ang II levels (16). Then, uAGT concentration is deemed to reflect the extension of renal damage in patients with CKD, including IgA nephropathy $(17,18)$.

For the first time, we demonstrated increased copeptin plasma levels, along with enhanced levels of $\mathrm{uAQP} 2$, in untreated IgAN patients, suggesting an increase of the intrarenal action of the neuropeptide in the disease. This increase seems to be specific for IgAN, and is seemingly modest in other forms of CKD. No studies have previously explored the excretion of UAQP2 in human glomerulonephritis. We assume that the enhanced $\mathrm{UAQP} 2$ excretion measured in $\operatorname{IgAN}$ patients could likely be the end result of a complex series of driving signals on the kidney expression and trafficking of the water channel protein, comprising the increased levels of AVP, the hyperactivity of renal RAS and, on the other side, the enhanced renal concentration of BK, as inferred by the increase of $\mathrm{uBK}$ recorded at the start of the study. It is worth noting that the increase of uBK mostly reflects the increase in the local concentration of the kinin, since BK serum levels of IgAN patients
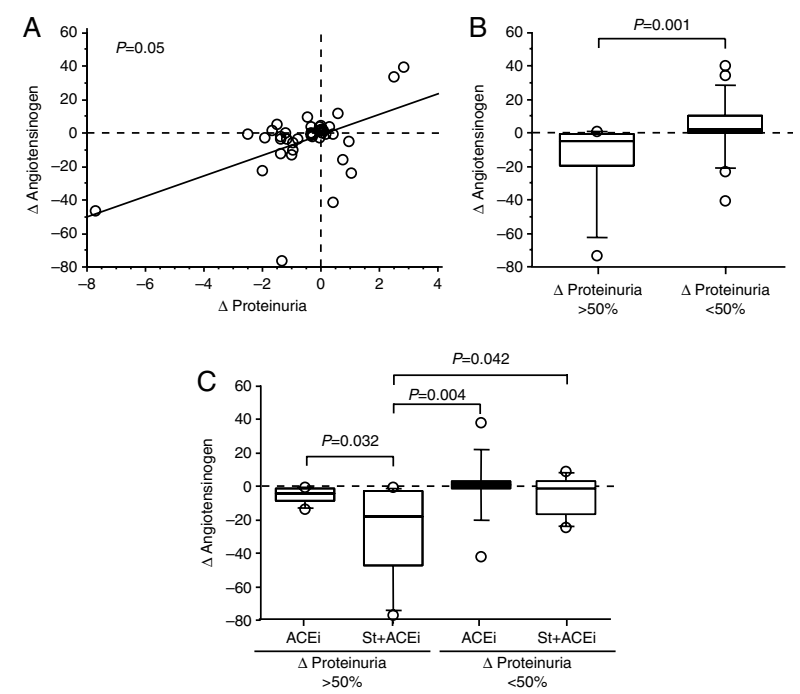

Figure $5(\mathrm{~A})$ Correlation between the change $(\Delta)$ of urinary angiotensinogen (AGT)) and the change $(\Delta)$ of $24 \mathrm{~h}$ proteinuria $(\mathrm{g} / \mathrm{die})$ ) in IgAN patients after 6-month treatment with either ACEi or steroids + ACEi (Spearman's rank correlation test). (B) Change of AGT urine excretion in patients, distinguished in responders $(>50 \%$ decrease of proteinuria) and non-responders ( $<50 \%$ decrease of proteinuria) (Mann-Whitney $U$ test). (C) Change of urinary AGT excretion according to the type of treatment and the modification of daily proteinuria (ANOVA test). Box-and-whisker plots, as in Fig. 1. 
Table 4 Association between the modification of urinary AQP2 and AGT and the change of daily proteinuria (g/die) after 6-month treatment with either $\mathrm{ACEi}$ or steroids $+\mathrm{ACEi}$.

\begin{tabular}{|c|c|c|c|c|c|c|c|c|}
\hline & \multicolumn{4}{|c|}{ Simple logistic model } & \multicolumn{4}{|c|}{ Multiple logistic model } \\
\hline & OR & $95 \% \mathrm{Cl}$ & $P^{*}$ & $R^{2}$ & OR & $95 \% \mathrm{Cl}$ & $P^{\star}$ & $R^{2}$ \\
\hline AQP2 (100 fmol/mg CrUr) & 1.21 & $1.05-1.39$ & $<0.0001$ & 0.338 & 1.2 & $1.01-1.42$ & & \\
\hline AGT (ng/mg CrUr) & 1.05 & $1.00-1.11$ & 0.0008 & 0.156 & 1.05 & $0.98-1.12$ & $<0.0001^{\dagger}$ & $0.46^{\dagger}$ \\
\hline
\end{tabular}

${ }^{*}$ likelihood ratio; ${ }^{\dagger}$ comparing AQP2 and AGT Cl levels.

were lower than in controls $(61.38 \pm 7.5$ vs 114.19 $\pm 28.57 \mathrm{pg} / \mathrm{ml} ; \quad P=0.04)$. Recent evidence suggests that AVP may play a role in the genesis and exacerbation of renal damage (5). The urinary excretion of AQP2 is taken to closely parallel changes in intrarenal AVP action. Thus, the increase of uAQP2, described here, would support a contributory role of AVP in the progression of renal damage of IgAN, and its levels of excretion are seemingly associated with the degree of deterioration of renal function.

We then found that the presence of arterial hypertension was associated with higher levels of uAGT and UAQP2 in IgAN patients, in the absence of any treatment (Fig. 2). As for uAGT, our findings confirm previous studies (19), and extend the correlation between uAGT and BP also to IgAN patients. Then, we demonstrate for the first time that UAQP2 associates with the presence of systemic hypertension in humans, similar to animal models (20).

Following 6-month treatment with ACEi alone, uAGT and $\mathrm{UAQP} 2$, on average, failed to show any significant modification (Fig. 3). At variance, the addition of steroids caused a significant decrease in the concentration of both the urine variables examined (Fig. 3A and B). As for the diminished AQP2 excretion, glucocorticoids exert a powerful inhibitory effect on hypothalamic regulatory pathways of AVP secretion $(21,22)$. Moreover, long-term aldosterone stimulation has been reported to decrease apical expression of AQP2 (23, 24). In other words, steroids can decrease AQP2 expression through both AVP-dependent and AVPindependent mechanisms. Then, the down-modulation of uAGT by steroids is rather puzzling. In cultured human tubular epithelial cells, glucocorticoids have been shown to activate AGT gene transcription (25). However, glucocorticoids would also down-regulate Ang II AT1 receptors (26), and in this way may dampen Ang II-induced stimulation of AGT expression by proximal tubular cells (27). Moreover, AGT transcription in IgAN correlates with tubulointerstitial inflammation (1), and steroids potently inhibit tissue inflammation. Whatever the mechanisms, our findings suggest that 6-month treatment with corticosteroids depresses intrarenal RAS activity more markedly than ACEi alone in IgAN.

Of note, the change in uAQP2 following drug therapies resulted to correlate with the change of uAGT, which would support a functional relationship between the two systems investigated, as suggested by in vitro models (8) and further supported by multivariate analysis (Table 3).

We found that patients with a higher decrease in proteinuria $(>50 \%)$, and therefore with a diminished risk of progression (28), exhibited a definitely higher drop of $\mathrm{UAQP} 2$ and uAGT (Figs 4B and 5B), regardless of the type of therapy done (Figs $4 \mathrm{C}$ and $5 \mathrm{C}$ ). Then, baseline levels of uAQP2 were strong predictors of patients' response to the therapy: the higher its levels at histological diagnosis, the higher its decrease following pharmacological treatments, and the higher the decrease of daily proteinuria.

Urinary AGT has been reported to significantly correlate with urinary protein in patients with CKD, including $\operatorname{IgAN}(17,18)$. Consequently, its decrease is expected to associate with a decrease in daily proteinuria. The association between the change in proteinuria and the change in AQP2 excretion is striking. Bardoux et al. (7) showed that stimulation of AVP V2 receptors induces a rise in urinary albumin excretion in humans, which is only partially prevented by ACEi in rats. In a large population study, plasma copeptin levels was shown to be associated with microalbuminuria, consistent with the hypothesis that AVP is involved in urinary albumin excretion (29). In a rat model of overt nephropathy, the administration of AVP receptor antagonist improved RAS blockers-induced amelioration of renal function and structure, and enhanced their antiproteinuric effect (30). Thus, the association between the decrease in proteinuria and the decrease in $\mathrm{UAQP} 2$ would reflect a down-regulation of the renal action of AVP, following steroid $(22,24)$ and/or ACEi treatment $(31,32)$.

In summary, the present exploratory study indicates that: i) IgAN patients at the time of histological diagnosis present with an increase in the urinary excretion of AQP2 and AGT, reflecting enhanced intrarenal AVP and RAS activity, as well as the severity of renal damage; ii) definitely higher levels of baseline UAQP2 are associated with the presence arterial hypertension and predict a positive treatment response and iii) the addition of steroids to ACEi enhances their antiproteinuric effect and causes a strong inhibition of uAGT and UAQP2 excretion. This suggests that steroids would heighten the inhibition of renal RAS activity 
exerted by ACEi, and interfere with AVP-dependent induction of renal damage.

Collectively, the above findings seem to support a role of AVP-AQP 2 axis, possibly interacting with the RAS, in the progression of $\operatorname{IgAN}$ and suggest AQP2 as a possible novel marker of IgAN and of disease progression.

On the other hand, we are aware of some limitations of this study preventing firm conclusions. First, we examined a relatively limited number of patients over a short time period, which precluded the use of hard end points, such as doubling of serum creatinine, and forced to the adoption of a surrogate marker of disease progression, namely daily proteinuria. Then, we failed to measure serum copeptin during and/or at the end of drug therapy. Finally, a conclusive and direct demonstration of the role of AVP-AQP2 axis on the progression of IgAN would require the administration of a selective AVP V2-receptor antagonist, such as tolvaptan, for relatively long periods.

\section{Declaration of interest}

The authors declare that there is no conflict of interest that could be perceived as prejudicing the impartiality of the research reported.

\section{Funding}

This study was supported by Cassa di Risparmio di Puglia Fundation and Ministry of Health (ex art 12; principal investigators Prof. L Gesualdo).

\section{Acknowledgements}

We acknowledge Dr Annamaria Di Palma (Department of Medical Biosciences, Division of Nephrology and Dialysis) for her clinical support in the histological examination, Dr Matilde Picozzi (Immunohematology and Transfusional Medicine Unit) for her contribution to serum collection of healthy volunteers and Dr Michele Falcone (Clinical Analysis Unit, Ospedali Riuniti) for his enthusiastic helpfulness in analysing bioclinical samples.

\section{References}

1 Del Prete D, Gambaro G, Lupo A, Anglani F, Brezzi B, Magistroni R, Graziotto R, Furci L, Modena F, Bernich P, Albertazzi A, D’Angelo A \& Maschio G. Precocious activation of genes of the reninangiotensin system and the fibrogenic cascade in IgA glomerulonephritis. Kidney International 200364 149-159. (doi:10. 1046/j.1523-1755.2003.00065.x)

2 Miyake-Ogawa C, Miyazaki M, Abe K, Harada T, Ozono Y, Sakai H, Koji T \& Kohno S. Tissue-specific expression of renin-angiotensin system components in IgA nephropathy. American Journal of Nephrology 200525 1-12. (doi:10.1159/000083224)

3 Dillon JJ. Angiotensin-converting enzyme inhibitors and angiotensin receptor blockers for IgA nephropathy. Seminars in Nephrology 200424 218-224. (doi:10.1016/j.semnephrol.2004.01.003)

4 Coppo R, Peruzzi L, Amore A, Piccoli A, Cochat P, Stone R, Kirschstein M \& Linné T. IgACE: a placebo-controlled, randomized trial of angiotensin-converting enzyme inhibitors in children and young people with IgA nephropathy and moderate proteinuria. Journal of the American Society of Nephrology 200718 1880-1888. (doi:10.1681/ASN.2006040347)
5 Bolignano D \& Zoccali C. Vasopressin beyond water: implications for renal diseases. Current Opinion in Nephrology and Hypertension 201019 499-504. (doi:10.1097/MNH.0b013e32833d35cf)

6 Bankir L. Antidiuretic action of vasopressin: quantitative aspects and interaction between V1a and V2 receptor-mediated effects. Cardiovascular Research 200151 372-390. (doi:10.1016/S00086363(01)00328-5)

7 Bardoux P, Bichet DG, Martin H, Gallois Y, Marre M, Arthus MF, Lonergan M, Ruel N, Bouby N \& Bankir L. Vasopressin increases urinary albumin excretion in rats and humans: involvement of V2 receptors and the renin-angiotensin system. Nephrology, Dialysis, Transplantation 2003 18 497-506. (doi:10.1093/ndt/18.3.497)

8 Li C, Wang W, Rivard CJ, Lanaspa MA, Summer S \& Schrier RW. Molecular mechanisms of angiotensin II stimulation on aquaporin-2 expression and trafficking. American Journal of Physiology. Renal Physiology 2011300 F1255-F1261. (doi:10.1152/ajprenal.00469.2010)

9 Wen H, Frokiaer J, Kwon TH \& Nielsen S. Urinary excretion of aquaporin-2 in rat is mediated by a vasopressin-dependent apical pathway. Journal of the American Society of Nephrology 199910 1416-1429.

10 Lee KSM, Rao VM, Franklin WA, Schiffer MS, Aronson AJ, Spargo BH \& Katz AI. IgA nephropathy: morphologic predictors of progressive renal disease. Human Pathology 198213 314-322. (doi:10.1016/S0046-8177(82)80221-9)

11 Valenti G, Laera A, Gouraud S, Pace G, Aceto G, Penza R, Selvaggi FP \& Svelto M. Low-calcium diet in hypercalciuric enuretic children restores AOP2 excretion and improves clinical symptoms. American Journal of Physiology. Renal Physiology 2002 283 F895-F903. (doi:10.1152/ajprenal.00354.2001)

12 Morgenthaler NG, Struck J, Alonso C \& Bergmann A. Assay for the measurement of copeptin, a stable peptide derived from the precursor of vasopressin. Clinical Chemistry 200652 112-119. (doi:10.1373/clinchem.2005.060038)

13 Jaspard E, Wei L \& Alhenc-Gelas F. Differences in the properties and enzymatic specificities of the two active sites of angiotensin I-converting enzyme (kininase II). Studies with bradykinin and other natural peptides. Journal of Biological Chemistry $1993 \mathbf{2 6 8}$ 9496-9503.

14 Tamma G, Carmosino M, Svelto M \& Valenti G. Bradykinin signaling counteracts cAMP-elicited aquaporin 2 translocation in renal cells. Journal of the American Society of Nephrology 200516 2881-2889. (doi:10.1681/ASN.2005020190)

15 Coppo R, Amore A, Gianoglio B, Cacace G, Picciotto G, Roccatello D, Peruzzi L, Piccoli G \& De Filippi PG. Angiotensin II local hyperreactivity in the progression of IgA nephropathy. American Journal of Kidney Diseases 199321 593-602.

16 Nishiyama A, Konishi Y, Ohashi N, Morikawa T, Urushihara M, Maeda I, Hamada M, Kishida M, Hitomi H, Shirahashi N, Kobori H \& Imanishi M. Urinary angiotensinogen reflects the activity of intrarenal renin-angiotensin system in patients with $\operatorname{IgA}$ nephropathy. Nephrology, Dialysis, Transplantation 201126 170-177. (doi:10.1093/ndt/gfq371)

17 Kim SM, Jang HR, Lee YJ, Lee JE, Huh WS, Kim DJ, Oh HY \& Kim YG. Urinary angiotensinogen levels reflect the severity of renal histopathology in patients with chronic kidney disease. Clinical Nephrology $2011 \quad 76 \quad 117-123 . \quad$ (doi:10.5414/CN 107045)

18 Yamamoto T, Nakagawa T, Suzuki H, Ohashi N, Fujigaki Y, Kato A, Nakamura Y, Suzuki F \& Hishida A. Urinary angiotensinogen as a marker of intrarenal angiotensin II activity associated with deterioration of renal function in patients with chronic kidney disease. Journal of the American Society of Nephrology $2007 \mathbf{1 8}$ 1558-1565. (doi:10.1681/ASN.2006060554)

19 Kobori H, Urushihara M, Xu JH, Berenson GS \& Navar LG. Urinary angiotensinogen is correlated with blood pressure in men (Bogalusa Heart Study). Journal of Hypertension $2010 \mathbf{2 8}$ 1422-1428. (doi:10.1097/HJH.Ob013e3283392673)

20 Buemi M, Nostro L, Di Pasquale G, Cavallaro E, Sturiale A, Floccari F, Aloisi C, Ruello A, Calapai G, Corica F \& Frisina N. 
Aquaporin-2 water channels in spontaneously hypertensive rats. American Journal of Hypertension 200417 1170-1178. (doi:10. 1016/j.amjhyper.2004.07.003)

21 Watts AG. Glucocorticoid regulation of peptide genes in neuroendocrine CRH neurons: a complexity beyond negative feedback. Frontiers in Neuroendocrinology 200526 109-130. (doi:10.1016/ j.yfrne.2005.09.001)

22 Bähr V, Franzen N, Oelkers W, Pfeiffer AF \& Diederich S. Effect of exogenous glucocorticoid on osmotically stimulated antidiuretic hormone secretion and on water reabsorption in man. European Journal of Endocrinology 2006155 845-848. (doi:10.1530/eje.1. 02299)

23 Arriza JL, Weinberger C, Cerelli G, Glaser TM, Handelin BL, Housman DE \& Evans RM. Cloning of human mineralocorticoid receptor complementary DNA: structural and functional kinship with the glucocorticoid receptor. Science 1987237 268-275. (doi:10.1126/science.3037703)

24 De Seigneux S, Nielsen J, Olesen ET, Dimke H, Kwon TH, Frøkiaer J \& Nielsen S. Long-term aldosterone treatment induces decreased apical but increased basolateral expression of AQP2 in CCD of rat kidney. American Journal of Physiology. Renal Physiology 2007293 F87-F99. (doi:10.1152/ajprenal.00431.2006)

25 de Haij S, Adcock IM, Bakker AC, Gobin SJ, Daha MR \& van Kooten C. Steroid responsiveness of renal epithelial cells. Dissociation of transrepression and transactivation. Journal of Biological Chemistry 2003278 5091-5098. (doi:10.1074/jbc.M209836200)

26 Chansel D, Llorens-Cortes C, Vandermeersch S, Pham P \& Ardaillou R. Regulation of angiotensin II receptor subtypes by dexamethasone in rat mesangial cells. Hypertension $1996 \mathbf{2 7} 867-874$.

27 Kobori H, Prieto-Carrasquero MC, Ozawa Y \& Navar LG. AT1 receptor mediated augmentation of intrarenal angiotensinogen in angiotensin II-dependent hypertension. Hypertension $2004 \mathbf{4 3}$ 1126-1132. (doi:10.1161/01.HYP.0000122875.91100.28)
28 Manno C, Torres DD, Rossini M, Pesce F \& Schena FP. Randomized controlled clinical trial of corticosteroids plus ACE-inhibitors with long-term follow-up in proteinuric IgA nephropathy. Nephrology, Dialysis, Transplantation 200924 3694-3701. (doi:10.1093/ndt/ gfp356)

29 Meijer E, Bakker SJ, Halbesma N, de Jong PE, Struck J \& Gansevoort RT. Copeptin, a surrogate marker of vasopressin, is associated with microalbuminuria in a large population cohort. Kidney International 201077 29-36. (doi:10.1038/ki. 2009.397)

30 Perico N, Zoja C, Corna D, Rottoli D, Gaspari F, Haskell L \& Remuzzi G. V1/V2 vasopressin receptor antagonism potentiates the renoprotection of renin-angiotensin system inhibition in rats with renal mass reduction. Kidney International $2009 \mathbf{7 6}$ 960-967. (doi:10.1038/ki.2009.267)

31 Santucci A, Leonetti Luparini R, Ferri C, Ficara C, Giarrizzo C \& Balsano F. Relationship between vasopressin and the reninangiotensin-aldosterone system in essential hypertension: effect of converting enzyme inhibitor on plasma vasopressin. Journal of Hypertension 19853 (Suppl.) S133-S134.

32 Armando I, Carranza A, Nishimura Y, Hoe KL, Barontini M, Terrón JA, Falcón-Neri A, Ito T, Juorio AV \& Saavedra JM. Peripheral administration of an angiotensin II AT(1) receptor antagonist decreases the hypothalamic-pituitary-adrenal response to isolation stress. Endocrinology 2001142 3880-3889. (doi:10.1210/en. 142.9.3880)

Received 9 June 2011

Revised version received 28 July 2011

Accepted 8 August 2011 